International Journal of Forensic Science \& Pathology (JJFP)

ISSN 2332-287X

\title{
Fatal Air Embolism Associated With Pneumatosis Cystoides Intestinalis
}

Soriano $\mathrm{BJ}^{1}$, Corliss $\mathrm{RF}^{1}$, Stier $\mathrm{MA}^{1 *}$

Case Report

Department of Pathology and Laboratory Medicine, University of Wisconsin School of Medicine and Public Health, 600 Highland Avenue, Madison, WI, USA.

Abstract
We report a unique fatality associated with pneumatosis cystoides intestinalis. The proposed mechanism of lethality is air emboliza-
tion. Our basis for this interpretation is autopsy exclusion of other lethal mechanisms in association with identifiable physical find-
ings. We offer anatomic and physiologic explanations for our interpretation, in association with a brief discussion thereof.
Key Words: Air Embolism; Pneumotosis Cystoides Intestinalis; Portal Venous Air Embolism; Diverticulitis; Pseudomembranous
Colitis.

\section{*Corresponding Author:}

Michael A Stier MD,

Department of Pathology and Laboratory Medicine,

University of Wisconsin School of Medicine and Public Health,

600 Highland Avenue, Madison, WI, USA.

E-mail: mastier@wisc.edu

Received: October 21, 2013

Accepted: November 25, 2013

Published: November 27, 2013

Citation: Soriano BJ, Corliss RF, Stier MA (2013) Fatal Air Embolism Associated with Pneumatosis Cystoides Intestinalis. Int J Forensic Sci Pathol. 1(2), 4-6. doi: http://dx.doi.org/10.19070/2332-287X-130002

Copyright: Stier $\mathbf{M A}{ }^{\odot}$ 2013. This is an open-access article distributed under the terms of the Creative Commons Attribution License, which permits unrestricted use, distribution and reproduction in any medium, provided the original author and source are credited.

\section{Introduction}

Venous air embolism is an infrequent but recognized lethal mechanism encountered in a variety of clinical settings. The most frequent is as a complication of physical trauma directly exposing atmospheric gas to the vascular system. In anticipation of this process, pre-autopsy radiography is considered the historical standard for establishing the diagnosis. There are, however, clinical scenarios in which air embolization is not predicted. This leads forensic specialists to forego the radiological studies. Despite this, the diagnosis of lethal air embolism may be possible. Our case exemplifies this theory.

\section{Case Report / History}

The patient was a 53-year-old male with his own sign-making and designing company. He denied tobacco use, and alcohol use was reported as an occasional beer. His medical history was significant for remote colon polyps, left ankle degenerative joint disease and childhood asthma. He more recently sustained a left knee injury from a chainsaw accident approximately three weeks prior to his death. This was complicated by cellulitis proximal to the wound and was treated with a two week course of Augmentin (amoxicillin/clavulanate). During antibiotic therapy, he developed diarrhea, abdominal cramping, low grade fever $\left(102^{\circ} \mathrm{F}\right)$, and nausea with some vomiting. His wife also reported that he was "swallowing a lot of air." Despite finishing the course of antibiotic treatment, his symptoms progressed. One week after completing therapy, his abdominal pain and discomfort were severe enough to prompt a visit to a local urgent care clinic. He was thereupon admitted directly for dehydration and presumed antibiotic associated enterocolitis.

On clinical exam, he appeared uncomfortable, writhing in bed and complaining of abdominal and rectal pain. His abdomen was distended and tense, with bilateral guarding and tenderness upon palpation. His left knee scar appeared well healed and without erythema or induration. The proximal medial thigh region in the area of prior cellulitis appeared completely resolved. Laboratory tests begun included Clostridium difficile stool antigen as well as stool and blood bacterial cultures. Abdominal and pelvic CT scans with contrast demonstrated diffuse thickening of the colonic wall with some relative sparing near the splenic flexure. In conjunction with the history, these findings suggested pseudomembranous colitis. Clinical features of severe sepsis were lacking, although he remained mildly febrile. On the evening of admission, he appeared more comfortable and with less distress. At this time, another physical examination was performed which included deep manual abdominal palpation. During this procedure he appeared acutely uncomfortable and stated he felt as though he were "hyperventilating." He subsequently became more anxious, pale and diaphoretic. His pulse was described as "thready." He was transferred to the intensive care unit were he became unresponsive. Resuscitative efforts, including intubation failed to yield results, and he was declared dead after 45 minutes in the intensive care unit. The following day, laboratory tests were cancelled and a postmortem exam was scheduled.

An autopsy was performed with a postmortem interval of less than 8 hours. Rigor mortis was in the onset phase, the body was ambient in cutaneous temperature, and livor mortis appeared dorsal and clearly blanching. No features of early decomposition were present and the remainder of the external examination was unremarkable. The thoracic examination showed a heart in the anticipated anatomic location without pericardial fluids. The epicardial veins were observed to have a near confluent intraluminal gas presence, alternating with a beaded distribution of bubbles. 
The right atrium and ventricle were pathologically distended and tense. Opening and observing the right heart chambers in situ revealed markedly foamy blood therein. The froth consisted of gaseous bubbles ranging in size from 0.1-0.6 centimeters, admixed with the cellular and fluid hematalogic components. The heart weighed 430 grams and was without additional pathologic findings. Lung weights were mildly increased at 470 and 520 grams, left and right, respectively.

The internal abdominal exam revealed aggregate gas filled vacuoles restricted to an approximate 10 centimeter segment of the descending colonic serosa. These measured 0.1-0.3 centimeters individually. There was an associated 1.7 liters of serous fluid in the peritoneal space, however, no gross indication of peritonitis was observed. Additionally, non-inflamed and intact appearing sigmoid diverticuli were present. The distal colonic mucosa displayed focal pseudomembrane formations.

The histological analysis of colonic sections showed numerous submucosal and mucosal cystic spaces without inflammation or specific intracystic contents. Elongated gram positive rods consistent with Clostridial bacteria were present in some areas of submucosa and lamina propria. Focal mixed inflammation was noted in some diverticula and focal, minor fibrin deposition was noted overlying intact, non-inflamed mucosa. The latter correlates with the gross observation of focal pseudomembrane formation. Microscopic sections of cardiopulmonary tissue were noncontributory.

Of note, the hepatic histoarchitecture showed mild distortion in a unique pattern. This finding consists of irregular, although localized expansion of sinusoidal margins. The edges of interface at these foci appeared somewhat scalloped, suggesting aggregates of gas bubbles. Portal triads revealed diffuse relative dilatation of portal venules without inflammation. No other relevant microscopic observations were made and the toxicological analysis was negative.

Given the anatomical findings in conjunction with the clinical history, the cause of death is attributed to acute portal venous air embolism due to pneumatosis cystoides intestinalis (PCI). The clinical course leading to death is linked to the unintentional chainsaw injury. Therefore, the assigned manner is accidental.

\section{Discussion}

The gold standard for diagnosis of fatal air embolism has been pre-autopsy chest radiography along with relevant anatomical findings (1-5). While we recognize the historic standard, anticipation of this potentially lethal process isn't always possible and pre-autopsy X-ray does not uniformly occur. We propose this case exemplifies an alternative constellation of findings allowing this diagnosis. This consists of the demonstrati0on of copious bubbly, frothy blood in right cardiac chambers, the absence of a significant competing acutely lethal disease process, a lack of gasforming decomposition, and the clinical history of relative rapid demise. Another element to consider in making this diagnosis is defining a viable source for gas to enter the circulatory system.

Fatal air embolism from pneumotosis cystoides intestinalis (PCI) appears to be exceedingly rare, but has been described (6). Its overall mortality is likely under recognized because of a variety of factors. PCI is characterized by multiple gas filled serosal or submucosal cysts involving portions of the gastrointestinal tract (10).
It is not uncommon, usually seen in association with necrotizing enterocolitis (NEC). While NEC carries a mortality rate of 30\% (7), its fatal mechanistic effects are presumed to be complications of bowel ischemia. Our case is without bowel necrosis and the mechanism of demise appears purely related to air embolization. Features of decomposition were lacking and despite the later report of Clostridial culture positivity, the autopsy findings are not that of a typical septic death with such a putrifying organism.

Other causes of PCI include chronic pulmonary disease, intestinal bypass surgery, inflammatory bowel disease, and several unrelated conditions ranging from trauma $(8,9)$ to neoplasia that compromise mucosal integrity. In these cases, luminal or extracorporeal gas is allowed a physical path for entry into the mucosa or submucosa. Another proposed mechanism for PCI is intramural microbial gas production. In our case, the final clinical presentation was due to presumed antibiotic associated enterocolitis. While the autopsy results show focal mucosal membrane formation, widespread inflammation or frank tissue necrosis is absent. Focal diverticulitis, however, is identified. The relative contribution of these differing anatomic findings to the clinical symptoms in undefined. A contribution of the reported aerophagia is also possible.

The clinical deterioration of our patient is temporally associated with deep abdominal palpation upon physical exam. The subsequent decline and death, despite previous improvement in clinical status, supports an acute process. Intraluminal gas could access mucosal and submucosal layers through an inflamed diverticulum or through mucosal denudation in areas of membrane formation. We believe PCI was present at the time of the patient's terminal hospitalization. The proposed mechanism of embolization is coalescence of intramural gas, with physical introduction into the portal venous system, possibly facilitated by abdominal palpation.

Anterograde migration through the anastomosing portal vasculature of the liver and ultimately to the right heart via hepatic vein and inferior vena cava is the pathway of embolization. In this context, the histopathologic findings in the liver are of interest. We interpret the dilated portal venules and foci of irregular sinusoidal contours as a possible manifestation of the gaseous emboli. Since this case, two additional autopsies with probable venous portal air embolization from other causes have been reviewed. All cases show some degree of relative dilatation of portal venules and focal irregular sinusoidal histoarchitecture. Because gas is a non-stainable entity, further histologic testing is not useful. Nonetheless, this constellation of microscopic findings may assist in the diagnostic consideration of air embolization through hepatic parenchyma.

\section{References}

[1]. Camps FE, Gradwohl RBH, Lucas BGB, et al. Gradwohl's Legal medicine. [Bristol] Chicago: Wright; distributed by Year Book Medical; 1976.

[2]. Di Maio DJ, Di Maio VJM. Forensic pathology. New York: Elsevier; 1989.

[3]. Dolinak D, Matshes EW, Lew EO. Forensic pathology: Principles and Practice. Amsterdam; Boston: Elsevier/Academic Press; 2005.

[4]. Knight B. Forensic Pathology. New York: Oxford University Press; 1991.

[5]. Spitz WU, Fisher RS. Spitz and Fisher's Medicolegal Investigation of Death: Guidelines for the Application of Pathology to Crime Investigation. Springfield, Ill., U.S.A.: C.C. Thomas; 1993.

[6]. Bonnell H, French SW. Fatal air embolus associated with pneumatosis cystoides intestinalis. Am J Forensic Med Pathol. 1982; 3:69-72.

[7]. Goyal SK, Balsam D. Pneumatosis Intestinalis. Available at: http://emedicine.medscape.com/article/371955-overview

[8]. Kelly BS, Jr., Meyers P, Choe KA, et al. Traumatic pneumatosis cystoides intestinalis with portal venous air embolism. J Trauma. 1997; 42:112-114. 
[9]. Shuck JM, Malan LJ, Hammar MD. Pneumatosis cystoides intestinals due to blunt abdominal trauma. J Trauma. 1974; 14:435-440.

[10]. Koss, L.G. Abdominal gas cysts (pneumatosis cystoides intestinorum hominis). Arch Pathol 1952; 53:523-549 